OPEN ACCESS

Edited by:

Martina de Zwaan, Hannover Medical School,

Germany

Reviewed by:

Sergueï O. Fetissov,

Université de Rouen, France

Martin Aigner,

Karl Landsteiner University of Health

Sciences, Austria

*Correspondence:

Carol Kan

carol.kan@kcl.ac.uk

Specialty section:

This article was submitted to

Psychosomatic Medicine,

a section of the journal

Frontiers in Psychiatry

Received: 25 October 2019

Accepted: 03 March 2020

Published: 17 March 2020

Citation:

Kan C, Eid L, Treasure $J$ and Himmerich H (2020) A Meta-Analysis of Dropout and Metabolic Effects of Antipsychotics in Anorexia Nervosa.

Front. Psychiatry 11:208.

doi: 10.3389/fpsyt.2020.00208

\section{A Meta-Analysis of Dropout and Metabolic Effects of Antipsychotics in Anorexia Nervosa}

\author{
Carol Kan*, Laura Eid, Janet Treasure and Hubertus Himmerich \\ Psychological Medicine, King's College London, London, United Kingdom
}

Background: Second-generation antipsychotics are often used off-label in the treatment of anorexia nervosa (AN) across the clinical spectrum. Patients with anorexia nervosa often cite concerns about metabolic effects, such as weight gain, as reasons for their reluctance to start or continue second-generation antipsychotics. Improving our understanding of the metabolic effect patients experience and reasons underlying their disinclination will enable us to build rapport and guide our clinical decisions. We therefore aimed to conduct a comprehensive review of dropouts, metabolic effects, and patient-reported outcomes associated with second-generation antipsychotic in people with AN.

Method: EMBASE, Medline, and PsycINFO were searched for all relevant studies published until 2019, and retrieved studies were assessed for eligibility as per predefined inclusion criteria. A random-effects meta-analysis was conducted to assess overall dropout rates.

Results: Of 983 citations retrieved, 21 studies met the inclusion criteria for the systematic review and 10 studies had appropriate data for meta-analysis. Using the random effects model, the pooled dropout rate in the intervention arm (95\% confidence interval) from psychopharmacological trials was 28\% (19 to 38\%) in people with AN. Personal reasons or factors associated with study were commonest reason for dropout, not adverse events or metabolic effects as hypothesized.

Conclusion: Compared to personal reasons, drug-related factors such as side effects seem to play a lesser role for the discontinuation of antipsychotic treatment under trial conditions. This suggests an urgent need to consider and fully examine potential individual and patient-related factors that influence dropout rates in psychopharmacological trials and treatment compliance in clinical settings.

Keywords: anorexia nervosa, dropout, antipsychotic, metabolic effect, meta-analysis 


\section{BACKGROUND}

Anorexia nervosa (AN) is a debilitating illness with a median duration of 7-10 years. Approximately 20\% of individuals with AN develop a severe and enduring form of the illness (1) and a 22 years follow-up study found that only $68.2 \%$ of participants with AN have recovered (2). Treating AN successfully is a challenge. Admissions have continued to rise exponentially in the UK (3), even though National Institute for Health and Care Excellence (NICE) recommended outpatient treatment as a first line of management since $2004(4,5)$.

Various forms of outpatient psychological therapies have been developed. A recent meta-analysis of 35 randomized controlled trials (RCTs) concluded that specialised AN treatment was not superior to standard treatment at follow-up [effect size $(95 \% \mathrm{CI})$ for i) for weight outcomes: $0.11(-0.04$ to $0.27)$ and ii) psychological outcomes: -0.001 (-0.11 to 0.11$)]$ (6). A recent Cochrane Review also reported that there is little or no difference between specialist inpatient care and active outpatient or combined inpatient and outpatient care in weight gain at 12 months after the start of treatment [standardised mean difference (SMD): -0.22 ( -0.49 to 0.05$)]$ (7).

Second-generation antipsychotics are often used off-label in the treatment of AN. For example, a recent self-reported survey of prescribing practice in the UK reported that psychotropic medications are commonly prescribed to a minority of children and adolescents with AN, with olanzapine being the most frequent psychotropic drug (8). The rationale for their use includes weight gain and symptom relief such as reducing anxious feelings and weight/shape concerns. The clinical effectiveness of antipsychotics for people with AN is, however, unclear at present. To date, there have been four meta-analyses examining this effect (9-12). None of the meta-analyses reported a statistically significant differences for weight or changes in body mass index (BMI) between antipsychotics and active control/ placebo groups.

The metabolic effects of second-generation antipsychotics are serious and wide-ranging. They have been associated with weight gain, dyslipidemia, hypertension, hyperglycemia, and type 2 diabetes in people with schizophrenia (13). Genetic vulnerability may be relevant in developing metabolic effects. A recent genome-wide association study reported significant genetic correlations between metabolic-related phenotypes with AN, with the authors encouraging a reconceptualization of AN as a metabo-psychiatric disorder (14). This concept is further supported by a meta-analysis of clinical studies showing that AN is associated with increased insulin sensitivity [effect size (95\% CI): 1.66 (0.79 to 2.54)].

Patients with AN often cite metabolic effects as reasons for their reservations towards second-generation antipsychotics (15). For example, weight gain is a desired side effect for AN from a clinician's perspective, but patients are often reluctant to start or continue with them. Drop-out rate in clinical trials is defined as the proportion of participants who begin, but do not complete the full course of recommended treatment. It is a useful metric in determining the tolerability of a treatment. We therefore conducted a meta-analysis on the drop-out rates from psychopharmacology trials with second-generation antipsychotics. It can serve as a proxy indicator of the likelihood of a patient with AN in continuing secondgeneration antipsychotics. We also explored self-reported side effect, as patient-reported outcomes provide a unique window in directly understanding patients' experience and guide clinical decision-making (16). The main aim of this review is therefore to conduct a comprehensive review of dropouts, metabolic effects, and patient-reported outcomes associated with secondgeneration antipsychotic in people with AN.

\section{METHOD}

\section{Eligibility Criteria}

Abstracts were considered eligible for full manuscript data extraction if the study met all the following criteria: i) they compared the treatment outcomes of second-generation antipsychotics for AN; ii) the design was RCTs, open label trials, or observational studies; and iii) published in English. Participants of any age and any sex, with a diagnosis of AN were considered. For the meta-analysis, only studies with information about the proportion of participants who begin but do not complete the full course of recommended treatment in the intervention arm were considered.

\section{Study Selection}

We searched the Embase (1985 to 2019), PsycInfo (2005 to 2019), and Medline (1982 to 2018), using Ovid Database. We also hand-screened reference lists of all included articles to identify additional studies that were not found during the initial search. Keywords used in the searches included, but not limited to "anorexia nervosa", "atypical antipsychotic", "second generation antipsychotic", "amisulpride", "aripiprazole", "asenapine", "brexpiprazole", "cariprazine", "clozapine", "lurasidone", "olanzapine", "paliperidone", "pimavanserin", "quetiapine", "remoxipride", "risperidone", "sertindole", "ziprasidone" (exhaustive list of keywords in Appendix 1).

\section{Data Extraction}

Using a standardized data extraction sheet, the following information (if available) was extracted and recorded for each study: authors; year of publication; country of origin; treatment setting; study design; duration of intervention; age; sex; total numbers of participants in each treatment group; drop out in intervention group; reasons for dropouts; adverse event reporting; duration of intervention and concurrent treatment.

Dropout was defined as the number of participants who started treatment and were defined as having dropped out according to the definition of the study. We did not distinguish the time point of the dropout, as participants can drop out prior to starting the intervention, during the intervention, and after completing the intervention but fail to attend follow-up. The metabolic effects considered included but not limited to weight/BMI, glycaemic control, electrocardiogram (ECG) reports, lipids profile, liver function tests, prolactin, and self-reported side effects. 


\section{Quality of Study}

To assess the quality of studies, the Cochrane Collaboration's tool for assessing risk of bias was applied to randomized trials and Risk Of Bias In Non-randomized Studies-of Interventions (ROBINS-I) assessment tool for non-randomized studies (17, 18). These include adequacy of study design (observational or open label trails or RCT with an adequate control group); recruitment of sample and control for cofounding variables, such as age, sex, socioeconomic status. A study was considered to be of high quality if the study design consists of a control group; consecutive or random sampling method was used; adequate blinding of participants and personnel; appropriate reporting of outcome variables.

\section{Statistical Analysis}

All statistical analyses were performed using the "Metafor" package (19) in open source software programme R. We first transformed the proportion data using the Freeman-Tukey (double arcsine) transformation. This approach is recommended for proportion data as it produces more stable estimates of corresponding sampling variances for the sampling distribution of proportions close to 0 or $1(20,21)$. The transformed proportions and corresponding sampling variances were used in the meta-analysis and then backtransformed using the equation derived by Miller (22) for ease of interpretation.

To estimate the average overall dropout rate from active treatment, a weighted pooled event rate was calculated ("escalc" function in "Metafor"). The meta-analysis was conducted using a random effects model. Given the likely significant differences in study design, a fixed-effects model was deemed inappropriate. Significance threshold was set at $\mathrm{p}<0.05$. A Cochran Q test was performed to assess between-study heterogeneity of effect size (23). Publication biases were investigated with visual inspection of the funnel plots and Begg's rank correlation tests for funnel plot asymmetry (24).

\section{RESULTS}

\section{Study Selection}

The literature search resulted in 983 studies (Figure 1). After reviewing their titles and abstracts, 51 studies met the inclusion criteria and were retrieved for full text. Of these, 33 studies were excluded from the systematic review as they did not meet the inclusion criteria. Upon closer inspection of the remaining 18 studies, two studies utilized the same study population $(25,26)$. The study with the main aim of reporting the findings of olanzapine in people with AN was selected (25). One study was published as an abstract and the journal is now defunct (27). We therefore did not have access to any information about sampling method, baseline clinical characteristics of the sample population and variables of interest. Five additional studies that fulfilled the inclusion criteria were found from searches of the reference lists of included articles. In total, 21 studies were included in the systematic review and summarised in Table 1.
11 studies were excluded from the meta-analysis because they did not report information about the proportion of participants in the intervention arm who did not complete the study. Raw data were therefore not available to generate a standardized effect size for dropout rates. This resulted in 10 datasets being included in the meta-analysis.

\section{Qualitative Summary}

Of the 21 datasets included in the systematic review, 11 were RCTs $(25,28,32-35,39,42,46,47), 5$ open label trials $(29,40-43$, 45), 1 prospective study (38) and 4 retrospective cohort studies $(31,36,44)$. Sample size varies substantially, with a range between 8 and 152. The median number of participants was 31 with an interquartile range of 18 and 39 . There were only 3 studies with a sample size over $100(106,152$, and 152 respectively). In addition, the majority of the studies included in the systemic review $(n=$ 10) have been conducted in USA, with the rest being in Canada only $(n=3)$, Canada/US $(n=1)$, Australia $(n=3)$, and Italy $(n=$ 4). In terms of sample populations, 6 studies focused on adolescents, with the mean age ranging from 13.7 to 17.1, while 15 studies focused on adults, with a mean age ranging from 20.5 to 38 . Of the 14 studies with information on gender, 6 studies recruited only females, while the proportion of females in the remaining 8 studies range between 88.9 and $97.0 \%$.

In terms of treatment setting, 6 studies were conducted in inpatient, 7 in outpatients, 1 in day-care, and 7 across all three settings. For concurrent treatment, 20 studies reported adequate information for extraction. 10 studies allowed for concurrent medication, mainly antidepressants and benzodiazepines, 3 studies combined psychotherapy with second generationantipsychotics and 6 studies was part of a comprehensive eating disorder treatment programme or its participants receive multidisciplinary team inputs which can include psychotherapy and meal support. One study recorded that no participants received psychotherapy or any other psychiatric treatment during the study. For second-generation antipsychotics as the study drug in the intervention group, 13 studies examined olanzapine, 4 quetiapine, 1 risperidone, 1 aripiprazole, 1 amisulpride, and 1 study compared olanzapine with aripiprazole. Their associated treatment duration ranges from a minimum of 2 weeks to a maximum of 20 weeks. Descriptive data from the datasets are summarized in Table 1.

For the meta-analysis, 7 were $\operatorname{RCT}(25,28,32,34,35,37,39)$ and 3 open label trials $(40,41,45)$. Total number of participants in the intervention group ranged between 6 and 75. The majority of the studies have been conducted in USA $(n=6)$, with the rest being in Canada only $(n=1)$, Canada/US $(n=1)$ and Australia $(n=2)$. Two studies focused on adolescents, with the mean age ranging from 16 to 17.1. 8 focused on adults, with a mean age ranging from 20.5 to 34 . In terms of second-generation antipsychotics as the study drug in the intervention group, 5 studies examined olanzapine, 4 quetiapine and 1 risperidone.

\section{Meta-Analysis}

The pooled dropout rate in the intervention arm was estimated to be $28 \%$ (95\% CI: 19 to $38 \%$; $\mathrm{p}<0.01)$ in a random effect model. Results were not statistically significant heterogeneous (Q: 11.96, 


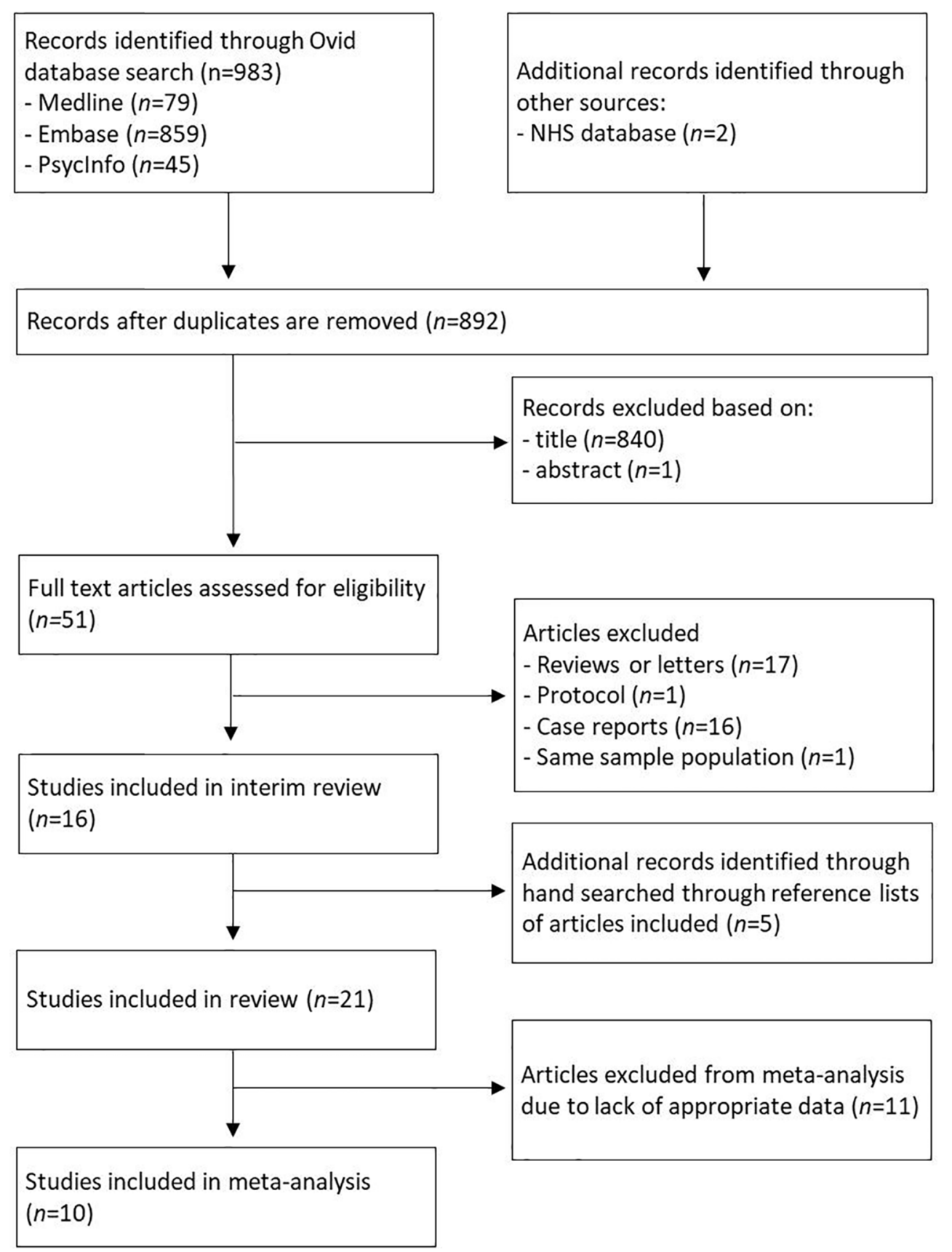

FIGURE 1 | Flow chart of studies included in systematic review.

df: 9, p: $\left.0.02, I^{2}: 33.1 \%\right)$. Visual inspection of the funnel plot suggested asymmetry, but the Begg's rank correlation test was statistically insignificant $(\tau: 0.27, \mathrm{p}: 0.28)$, indicating no publication bias.

\section{Outcome of Interest: Dropout}

Ten studies specified reasons for dropout. The percentage of each dropout reason, as a proportion of all the reasons given, are described below from most prevalent to least prevalent. The commonest reason for dropout was personal choices (43.5\%; 27/62), such as participants' voluntarily withdrawal from a study or preference of a different intervention. This was closely followed by study reasons, for example being lost to follow-up or failure to adhere to protocol $(25.8 \% ; 16 / 62)$. Of interest, $16.1 \%(10 / 62)$ of dropouts were due to poor response to the intervention or deterioration in mental state, with 6 
TABLE 1 | Summary table of primary studies included in the systemic review.

\begin{tabular}{|c|c|c|c|c|c|c|c|c|c|c|c|}
\hline Author & Setting & Study type & $\begin{array}{l}\text { Adults/ } \\
\text { Adolescent } \\
\text { Age mean (SD) }\end{array}$ & $\begin{array}{l}\% \text { of } \\
\text { female }\end{array}$ & $\begin{array}{l}\text { Number of } \\
\text { participants }\end{array}$ & $\begin{array}{l}\text { Dropout in } \\
\text { intervention } \\
\text { group }\end{array}$ & $\begin{array}{l}\text { Reasons } \\
\text { for dropout }\end{array}$ & Adverse event & $\begin{array}{l}\text { Intervention } \\
\text { dosage mean } \\
\text { (SD) }\end{array}$ & $\begin{array}{l}\text { Duration of } \\
\text { intervention } \\
\text { (weeks) }\end{array}$ & $\begin{array}{l}\text { Concurrent } \\
\text { treatment }\end{array}$ \\
\hline${ }^{*}$ Attia (28) & $\begin{array}{l}\text { US/Canada; } \\
\text { Outpatient }\end{array}$ & $\mathrm{RCT}$ & $\begin{array}{l}\text { Adults } \\
\text { Cx: } 30.0(11.0) \\
\text { Ix: } 28.0(10.9)\end{array}$ & $\begin{array}{c}96 \\
(146)\end{array}$ & $\begin{array}{l}\text { Cx: } 77 \\
\text { Ix: } 75\end{array}$ & $34 / 75$ & $\begin{array}{l}\text { Hospitalisation: } 5 \\
\text { Side effect: } 4 \\
\text { Suicidal ideation: } 3 \\
\text { Other: } 1 \\
\text { Voluntarily } \\
\text { withdrawal: } 12 \\
\text { Lost to follow-up: } 9\end{array}$ & $\begin{array}{l}\text { Hospitalisation: } 5 \\
\text { Suicidal ideation: } 3\end{array}$ & $\begin{array}{l}\text { Olanzapine } \\
7.77(1.07)\end{array}$ & 16 & $\begin{array}{l}\text { Psychotropics } \\
\text { ( } 41.4 \% \text { in sample) }\end{array}$ \\
\hline $\begin{array}{l}\text { Spettigue } \\
\text { (29) }\end{array}$ & $\begin{array}{l}\text { Canada; } \\
\text { Mainly } \\
\text { Inpatient }\end{array}$ & Open label trial & $\begin{array}{l}\text { Adolescents } \\
15.48(1.45)\end{array}$ & $\begin{array}{l}90.6 \\
(29)\end{array}$ & $\begin{array}{l}\text { At baseline, } \\
\text { Cx: 18; Ix: } 14 \\
8 \text { participants } \\
\text { switched to Ix } \\
\text { At end of } \\
\text { study, } \\
\text { Cx: 10; Ix: } 22\end{array}$ & NA & $\begin{array}{l}\text { Incapable to } \\
\text { participate: } 1 \\
\text { Unable to adhere to } \\
\text { protocol: } 1 \\
\text { Preferred a different } \\
\text { Ix: } 4\end{array}$ & None & $\begin{array}{l}\text { Olanzapine } \\
5.28 \text { (NA) }\end{array}$ & 12 & $\begin{array}{l}\text { Individual and family } \\
\text { therapy }\end{array}$ \\
\hline Frank (30) & $\begin{array}{l}\text { US; Inpatient/ } \\
\text { partial } \\
\text { inpatient }\end{array}$ & $\begin{array}{l}\text { Retrospective } \\
\text { chart review }\end{array}$ & $\begin{array}{l}\text { Adolescents } \\
\text { Cx: } 14.4(2.5) \\
\text { Ix: } 15.0(2.2)\end{array}$ & NA & $\begin{array}{l}\text { Cx: } 84 \\
\text { Ix: } 22\end{array}$ & NA & NA & NA & $\begin{array}{l}\text { Aripiprazole } \\
3.59(1.85)\end{array}$ & Minimal 3 & $\begin{array}{l}\text { Specialized eating } \\
\text { disorders } \\
\text { program }\end{array}$ \\
\hline Marzola (31) & Italy Inpatient & $\begin{array}{l}\text { Retrospective } \\
\text { chart review }\end{array}$ & $\begin{array}{l}\text { Adults } \\
25.43(9.4)\end{array}$ & $\begin{array}{l}90.7 \\
(68)\end{array}$ & $\begin{array}{l}\text { Cx: } 25 \\
\text { Ix-A: } 23 \\
\mid x-B: 27\end{array}$ & NA & NA & None & $\begin{array}{l}\text { Ix-A: } \\
\text { Aripiprazole } \\
9.13(6.33) \\
\text { Ix-B: Olanzapine } \\
6.11(3.27)\end{array}$ & $\begin{array}{l}\text { Mean: } 4.96 \\
(1.62)\end{array}$ & Antidepressants \\
\hline *Powers (32) & US; Outpatient & $\mathrm{RCT}$ & $\begin{array}{l}\text { Adults } \\
34(13.48)\end{array}$ & $\begin{array}{l}93.3 \\
(14)\end{array}$ & $\begin{array}{l}\text { Cx: } 9 \\
\mid x: 6\end{array}$ & $2 / 6$ & Hospitalisation: 2 & Hospitalisation: 2 & $\begin{array}{l}\text { Quetiapine } \\
177.7(90.8)\end{array}$ & 8 & NA \\
\hline Attia (33) & $\begin{array}{l}\text { US/Canada; } \\
\text { Outpatient }\end{array}$ & $\mathrm{RCT}$ & $\begin{array}{l}\text { Adults } \\
27.7(9.1)\end{array}$ & $\begin{array}{l}95.7 \\
(22)\end{array}$ & $\begin{array}{l}\text { Cx: } 12 \\
\text { Ix: } 11\end{array}$ & NA & NA & None & $\begin{array}{l}\text { Olanzapine } \\
7.95(2.7)\end{array}$ & 8 & $\begin{array}{l}\text { Antidepressants; no } \\
\text { psychological therapy }\end{array}$ \\
\hline $\begin{array}{l}\text { *Hagman } \\
\text { (34) }\end{array}$ & $\begin{array}{l}\text { US; Inpatient, } \\
\text { outpatient and } \\
\text { daycare }\end{array}$ & RCT & $\begin{array}{l}\text { Adolescents } \\
16(N A)\end{array}$ & $\begin{array}{l}100 \\
(40)\end{array}$ & $\begin{array}{l}\text { Cx: } 22 \\
\text { Ix: } 19\end{array}$ & $3 / 19$ & $\begin{array}{l}\text { Requested active Ix: } 1 \\
\text { Reasons unrelated to } \\
\text { study: } 2\end{array}$ & None & $\begin{array}{l}\text { Risperidone } \\
2.5(1.2)\end{array}$ & $\begin{array}{l}\text { Mean: } 8.6 \\
(4.2)\end{array}$ & $\begin{array}{l}\text { Antidepressants } \\
\text { (40\% in sample) }\end{array}$ \\
\hline $\begin{array}{l}\text { *Kafantaris } \\
\text { (35) }\end{array}$ & $\begin{array}{l}\text { US;Inpatient, } \\
\text { outpatient and } \\
\text { daycare }\end{array}$ & $\mathrm{RCT}$ & $\begin{array}{l}\text { Adolescents } \\
17.1 \text { (NA) }\end{array}$ & $\begin{array}{l}100 \\
(20)\end{array}$ & $\begin{array}{l}\text { Cx: } 10 \\
\text { Ix: } 10\end{array}$ & $3 / 10$ & $\begin{array}{l}\text { Sedation: } 1 \\
\text { Stopped Ix: } 2\end{array}$ & None & $\begin{array}{l}\text { Olanzapine } \\
8.5(N A)\end{array}$ & 10 & $\begin{array}{l}\text { Comprehensive } \\
\text { eating disorders } \\
\text { programme; no } \\
\text { group/family therapy }\end{array}$ \\
\hline Norris (36) & $\begin{array}{l}\text { Canada; } \\
\text { Inpatient, } \\
\text { outpatient and } \\
\text { daycare }\end{array}$ & $\begin{array}{l}\text { Retrospective } \\
\text { cohort study }\end{array}$ & $\begin{array}{l}\text { Adolescents } \\
\text { Cx: } 14.8(1.6) \\
\text { Ix: } 14.4(1.9)\end{array}$ & $\begin{array}{l}100 \\
(86)\end{array}$ & $\begin{array}{l}\text { Cx: } 43 \\
\text { Ix: } 43\end{array}$ & NA & NA & None severe & $\begin{array}{l}\text { Olanzapine } \\
5.0(3.75-7.5)\end{array}$ & $\begin{array}{l}\text { Minimal } 2 \\
\text { In subgroup }\end{array}$ & $\begin{array}{l}\text { Antidepressants } \\
(39 \%)+/- \\
\text { benzodiazepine (7\%) }\end{array}$ \\
\hline${ }^{*}$ Court (37) & $\begin{array}{l}\text { Australia; } \\
\text { Outpatient and } \\
\text { inpatient if } \\
\text { needed during } \\
\text { stay }\end{array}$ & $\begin{array}{l}\text { Open label } \\
\text { RCT }\end{array}$ & $\begin{array}{l}\text { Adults } 22.53 \\
\text { (NA) }\end{array}$ & $\begin{array}{l}97.0 \\
(32)\end{array}$ & $\begin{array}{l}\text { Cx: } 18 \\
\text { Ix: } 15\end{array}$ & $5 / 15$ & $\begin{array}{l}\text { Withdrawn consent: } 1 \\
\text { Adverse event: } 1 \\
\text { Side effect: } 1 \\
\text { Non-response: } 1 \\
\text { Failure to attend: } 1\end{array}$ & None severe & $\begin{array}{l}\text { Quetiapine } \\
322.5 \text { (NA) }\end{array}$ & 12 & $\begin{array}{l}\text { Antidepressants +/- } \\
\text { benzodiazepine }\end{array}$ \\
\hline Leggero (38) & $\begin{array}{l}\text { Italy; } \\
\text { Outpatient }\end{array}$ & $\begin{array}{l}\text { Prospective } \\
\text { cohort study }\end{array}$ & $\begin{array}{l}\text { Adolescents } \\
13.7(2.3)\end{array}$ & NA & $\mid x: 13$ & NA & NA & NA & Olanzapine & Unclear & $\begin{array}{l}\text { Multimodal including } \\
\text { psychotherapy, } \\
\text { assisted feeding, } \\
\text { psychoeducation, }\end{array}$ \\
\hline
\end{tabular}




\begin{tabular}{|c|c|c|c|c|c|c|c|c|c|c|c|}
\hline Author & Setting & Study type & $\begin{array}{l}\text { Adults/ } \\
\text { Adolescent } \\
\text { Age mean (SD) }\end{array}$ & $\begin{array}{l}\% \text { of } \\
\text { female }\end{array}$ & $\begin{array}{l}\text { Number of } \\
\text { participants }\end{array}$ & $\begin{array}{l}\text { Dropout in } \\
\text { intervention } \\
\text { group }\end{array}$ & $\begin{array}{l}\text { Reasons } \\
\text { for dropout }\end{array}$ & Adverse event & $\begin{array}{l}\text { Intervention } \\
\text { dosage mean } \\
\text { (SD) }\end{array}$ & $\begin{array}{l}\text { Duration of } \\
\text { intervention } \\
\text { (weeks) }\end{array}$ & $\begin{array}{l}\text { Concurrent } \\
\text { treatment }\end{array}$ \\
\hline & & & & & & & & & & & $\begin{array}{l}\text { and prolonged } \\
\text { control of somatic } \\
\text { conditions }\end{array}$ \\
\hline *Bissada (39) & $\begin{array}{l}\text { Canada; } \\
\text { Daycare }\end{array}$ & RCT & $\begin{array}{l}\text { Adults } \\
\text { Cx: } 29.67 \\
(11.59) \\
\text { Ix: } 23.61(6.50)\end{array}$ & $\begin{array}{l}100 \\
(34)\end{array}$ & $\begin{array}{l}\text { Cx:18 } \\
\mid x: 16\end{array}$ & $2 / 16$ & $\begin{array}{l}\text { Discontinued } \\
\text { daycare: } 1 \\
\text { Stopped Ix: } 1\end{array}$ & None & $\begin{array}{l}\text { Olanzapine } \\
6.61(2.32)\end{array}$ & 10 & $\begin{array}{l}\text { Comprehensive day } \\
\text { programme } \\
\text { Including group } \\
\text { therapy }\end{array}$ \\
\hline $\begin{array}{l}\text { Brambilla } \\
\text { (25) }\end{array}$ & $\begin{array}{l}\text { Italy; } \\
\text { Outpatient }\end{array}$ & RCT & $\begin{array}{l}\text { Adults } \\
\text { Cx } 26.3 \text { (8.5) }\end{array}$ & $\begin{array}{l}100 \\
(30)\end{array}$ & $\begin{array}{l}\text { Cx: } 15 \\
\text { Ix: } 15\end{array}$ & NA & NA & NA & $\begin{array}{l}\text { Olanzapine } \\
7.77(1.07)\end{array}$ & 12 & CBT \\
\hline $\begin{array}{l}\text { "Bosanac } \\
(40)\end{array}$ & $\begin{array}{l}\text { Australia; } \\
\text { Inpatient/ } \\
\text { outpatient }\end{array}$ & Open label trial & $\begin{array}{l}\text { Adults } \\
33.25(7.65)\end{array}$ & $\begin{array}{l}100 \\
(8)\end{array}$ & |x: 8 & $3 / 8$ & $\begin{array}{l}\text { Own request: } 1 \\
\text { Substance misuse: } 2\end{array}$ & None & $\begin{array}{l}\text { Quetiapine } \\
520(277.49)\end{array}$ & 8 & $\begin{array}{l}\text { Specialised and } \\
\text { multidisciplinary } \\
\text { treatment }\end{array}$ \\
\hline${ }^{*}$ Powers (41) & US; Outpatient & Open label trial & $\begin{array}{l}\text { Adults } \\
26.8(11.2)\end{array}$ & $\begin{array}{l}94.7 \\
(18)\end{array}$ & |x: 19 & $5 / 19$ & $\begin{array}{l}\text { Unknown: } 1 \\
\text { Seek treatment } \\
\text { elsewhere: } 1 \\
\text { Weight gain: } 1 \\
\text { Moved out of area: } 1 \\
\text { Non-response: } 1\end{array}$ & None severe & $\begin{array}{l}\text { Quetiapine } \\
150-300 \text { (NA) }\end{array}$ & 10 & None \\
\hline $\begin{array}{l}\text { Mondraty } \\
\text { (42) }\end{array}$ & $\begin{array}{l}\text { Australia; } \\
\text { Inpatient }\end{array}$ & RCT & $\begin{array}{l}\text { Adults } \\
25.3 \text { (NA) }\end{array}$ & NA & $\begin{array}{l}\text { Cx: } 7 \\
\text { Ix: } 8\end{array}$ & NA & NA & NA & $\begin{array}{l}\text { Cx: } \\
\text { Chlorpromazine } \\
50 \text { (NA) } \\
\text { Ix: Olanzapine } \\
10 \text { (NA) }\end{array}$ & $\begin{array}{l}\text { Mean: } \\
\text { (Unit: days) } \\
\text { Cx: } 53 \\
\text { (26) } \\
\text { Ix: } 46 \\
\text { (31) }\end{array}$ & $\begin{array}{l}\text { Antidepressants } \\
\text { (63\% in Ix arm) }\end{array}$ \\
\hline $\begin{array}{l}\text { *Barbarich } \\
\text { (43) }\end{array}$ & $\begin{array}{l}\text { US; Inpatient \& } \\
\text { outpatient }\end{array}$ & Open label trial & $\begin{array}{l}\text { Adults } \\
20.5(5.1)\end{array}$ & NA & Ix: 17 & $5 / 17$ & NA & NA & $\begin{array}{l}\text { Olanzapine } \\
4.7(1.6)\end{array}$ & 6 & $\begin{array}{l}\text { CBT and DBT mainly, } \\
\text { with } 1 \text { participant } \\
\text { being on } \\
\text { antidepressants }\end{array}$ \\
\hline Malina (44) & US; Inpatient & $\begin{array}{l}\text { Retrospective } \\
\text { cohort study }\end{array}$ & $\begin{array}{l}\text { Adults } \\
22(7)\end{array}$ & NA & Ix: 18 & NA & NA & NA & $\begin{array}{l}\text { Olanzapine } \\
4.7(2.4)\end{array}$ & $\begin{array}{l}\text { Range: } \\
17-20\end{array}$ & $\begin{array}{l}\text { Antidepressants, } \\
\text { benzodiazepine }\end{array}$ \\
\hline *Powers (45) & US; Outpatient & Open label trial & $\begin{array}{l}\text { Adults } \\
26.8(12.3)\end{array}$ & $\begin{array}{l}88.9 \\
(16)\end{array}$ & 18 & $4 / 18$ & Hospitalisation: 1 & NA & $\begin{array}{l}\text { Olanzapine } \\
10 \text { (NA) }\end{array}$ & 10 & $\begin{array}{l}\text { Psychotherapy for } 1 \\
\text { participant } \\
\text { and benzodiazepine } \\
\text { for } 2 \text { participants }\end{array}$ \\
\hline $\begin{array}{l}\text { Ruggiero } \\
\text { (46) }\end{array}$ & Italy; Inpatient & $\begin{array}{l}\text { Single blind } \\
\text { RCT }\end{array}$ & $\begin{array}{l}\text { Adults } \\
\text { Cx-A: } \\
23.69(4.57) \\
\text { Cx-B: } \\
24.5(50.6) \\
\text { Ix: } 24.33(5.76)\end{array}$ & NA & $\begin{array}{l}\text { Cx-A: } 13 \\
\text { Cx-B: } 10 \\
\text { Ix: } 12\end{array}$ & NA & NA & NA & $\begin{array}{l}\text { Cx-A: } \\
\text { Clomipramine } \\
57.69(25.79) \\
\text { Cx-B: } \\
\text { Fluoxetine } \\
28 \text { (10.32) } \\
\text { Ix: Amisulpride } \\
50 \text { (NA) }\end{array}$ & 12 & $\begin{array}{l}\text { Weight gaining } \\
\text { programme }\end{array}$ \\
\hline
\end{tabular}




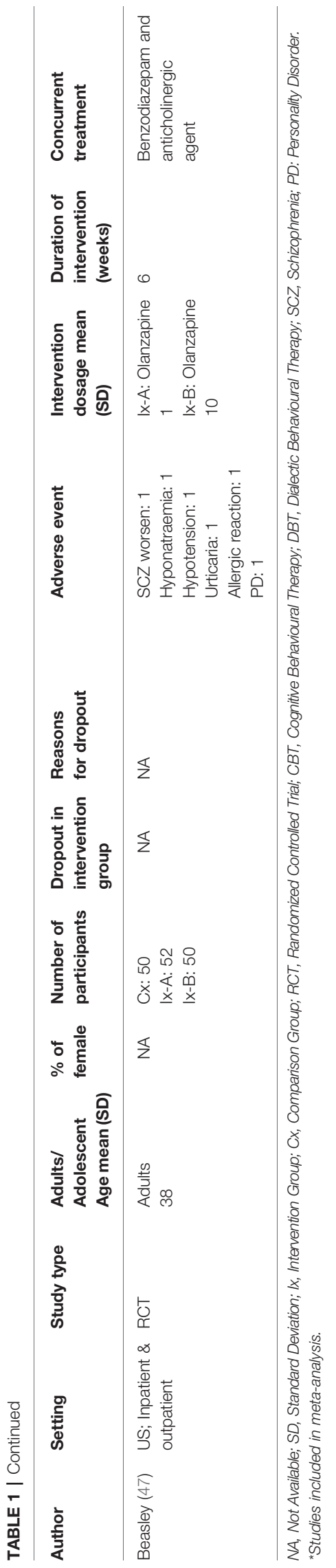

participants requiring hospitalization during the study. Adverse events were cited as a reason for dropouts in only $12.9 \%$ cases, with weight gain being mentioned once. There were, however, three incidences of suicidal ideations $(4.8 \%)$ as a reason for dropout from one study (28).

\section{Outcome of Interest: Metabolic Effects}

All studies included in the systematic review provided some information for weight/BMI/ideal body weight. This information was, however, difficult to interpret due to heterogeneity between studies. This included different duration of intervention and measures of weight, with some focus on weight gain over time or changes in pre- and postintervention BMI between groups. In addition, the handling of missing data analysis was often not discussed, leading it hard to summarize the results in a systematic manner. In brief, 6 studies reported a statistically difference between intervention and comparison groups in either weight/BMI increase over time $(28-30,33,36,47)$ whereas 9 studies concluded the opposite $(25,32,34,46,47)$. Six studies focused on the intervention group only, with 4 reporting a statistically significant weight gain/BMI increase or a greater proportion reaching their ideal body weight $(38,40,43,45)$. One study did not observe any statistically significant change in weight between baseline and end of treatment (41) while one study did not report any significance testing (44).

For glycaemic control, seven studies reported their findings. One study reported a statistically significant increase in fasting glucose in the intervention group (olanzapine) between baseline and end of study (35) whereas one study reported that 4 participants reported hypoglycaemic events with olanzapine (42). For ECG results, four studies reported their findings. One study reported that QTc prolongation was observed in one participant from both intervention (olanzapine) and comparison groups (29) whereas another study reported that one participant developed borderline QTc prolongation with olanzapine (31).

For other routine metabolic effects, there was limited information, with some studies stating that no abnormalities were observed during routine laboratory investigation. In summary, 5 studies reported lipid profiles, 5 for liver function test, and 2 on prolactin. Most studies did not report any statistically significant difference between intervention and comparison groups. One study reported that 3 participants developed raised cholesterol and one participant developed raised low-density lipoprotein in the intervention group (olanzapine) during the study period (31). One study concluded that more participants in the intervention group (olanzapine) experienced clinically significant abnormalities in lipid profiles, liver function tests and prolactin than comparison group, but the differences did not reach statistical difference (29). Another study reported that there is statistically difference in prolactin level between intervention (risperidone) and comparison groups (34), while another study reported that two participants in one study developed raised liver enzymes with olanzapine (38). 


\section{Outcome of Interest: Patients Reported Physical Side Effects}

Second-generation antipsychotics are associated with a range of physical side effects which may not be captured by biometric investigations. All studies included in the systemic review were examined for any description of patient reported physical side effects. In brief, 14/21 (67\%) studies documented physical side effects or adverse events. There was no information on the duration of the side effects, except for two studies describing them as mainly transient $(37,41)$.

In the intervention group, the most common side effect was sedation which was reported in 12 out of the 21 studies (57\%). This was followed by dizziness ( $\mathrm{n}=7$ studies, $33 \%)$, headache $(\mathrm{n}=$ $7,33 \%)$, gastrointestinal problems $(n=6,29 \%)$, insomnia $(n=5$, $24 \%)$, fatigue ( $\mathrm{n}=4,19 \%)$, muscular problems $(\mathrm{n}=4,19 \%)$. A few studies also reported dry mouth $(\mathrm{n}=3,14 \%)$, blurred vision $(\mathrm{n}=$ $2,10 \%)$, poor concentration $(\mathrm{n}=2,10 \%)$, agitation $(\mathrm{n}=2,10 \%)$, respiratory problems $(n=2,10 \%)$, lower extremity oedema $(n=2$, $10 \%)$, difficulty in staying still $(\mathrm{n}=1,5 \%)$, paraesthesia $(\mathrm{n}=1$, $5 \%)$, and dental problems $(n=1,5 \%)$. Of interest, participants who received placebo also reported physical side effects $(n=3$, $14 \%)(28,29,34)$. These included troubles concentrating, subjective restlessness, agitation, sleep problems, headache, constipation, dizziness, muscle stiffness, somnolence, dry mouth, fatigue, dizziness, gastrointestinal complaints, and headache.

\section{Risk of Bias and Strength of Evidence Quality}

For randomized trials, two studies did not blind participants and personal adequately $(37,42)$. For non-randomized trial, six studies did not have a comparison group as they were observation studies $(25,38,40,44,45)$. Other sources of bias were which could potentially threaten the external and internal validity of the findings include heterogeneous demographics across groups during baseline, a short duration of treatment, wide range of medication dosage, or confounding variables such as concurrent treatment. In addition, sample sizes of each individual studies were generally small and of the 21 studies included in the systematic review, only 10 studies reported appropriate dropout rates for the meta-analysis, further reducing the sample sizes. The challenges of small sample size were further compounded by the significant dropout observed and only missing data analysis was often not clearly detailed. The overall risk of bias was therefore low to medium (Figure 2).

\section{DISCUSSION}

To our knowledge, this is the first systematic review and metaanalysis of dropout associated with second-generation antipsychotics in people with AN. Our finding of $28 \%$ dropout rate (95\% CI: 19 to $38 \%$ ) from psychopharmacological trial is low when compared to $48.1 \%$ reported in a meta-analysis of secondgeneration antipsychotics in people with schizophrenia or schizoaffective disorder (48). Our dropout rate is, however, high when compared to psychotherapy. Meta-analyses have reported dropout rates of $24 \%$ for cognitive behaviour therapy in people eating disorders (49), 18.7\% for interpersonal therapy in eating disorders (50) and 19.9\% for individual psychotherapy in unipolar depression (51). Of interest, personal choices and factors associated with individual trials are the main reasons for dropout, not metabolic effects such as weight gain as initially hypothesised. Thus, our findings prompt the need to consider how specific patient and study characteristics may influence dropout rates in psychopharmacological trials in people with AN.

It is important to interpret our findings in the context of difficulties of recruiting and retaining participants with $\mathrm{AN}$ in psychopharmacological studies. For example, in a study of quetiapine, only $10.4 \%$ of the adult patients $(21 / 217)$ consented to treatment (32). A study in adolescents examining risperidone was ended after 4 years due to slow recruitment and exhausted funding (34). Thus, the extent of generalizability of our findings to those who declined to participate is at present unclear.

\section{Limitations}

A major source of heterogeneity is study design, given only 11 out of the 21 studies included in the systematic review were RCT in design. Another source of heterogeneity is concurrent treatment and study setting. The regimen of the secondgeneration antipsychotics also varies substantially between and within studies. The dosage is often flexible and individualtailored within studies. A major limitation of our review is that most studies included in the systematic review (67\%) and metaanalysis (80\%) were from USA/Canada, rendering it difficult to generalize our findings. In addition, most studies were small in size. Another major limitation of our review is that most studies included were small in size. This is further compounded by the significant proportion of participants who did not complete a study. Thus, adopting the "intention-to-treat" in data analysis according to the treatment groups being assigned at randomization, with appropriate handling of missing data is important. In addition, the long-term metabolic effects of second-generation antipsychotics in people with $\mathrm{AN}$ is currently unclear, as only two studies discussed follow-up beyond 3 months $(37,38)$.

\section{Clinical Implications}

Recruitment and retainment rates are a major challenge for psychopharmacology studies in AN. To encourage patients to consider second-generation antipsychotics as part of their treatment plan, it is important to explain the potential side effects experienced and stress the transient nature. Given that adverse events such as suicidal ideation were observed (28), it is important that patients are reviewed after being commenced on second-generation antipsychotics and address any side effects experienced promptly. Including family members in discussions about treatment and potential risks can allow for a more balanced appraisal of possible costs and benefits. 

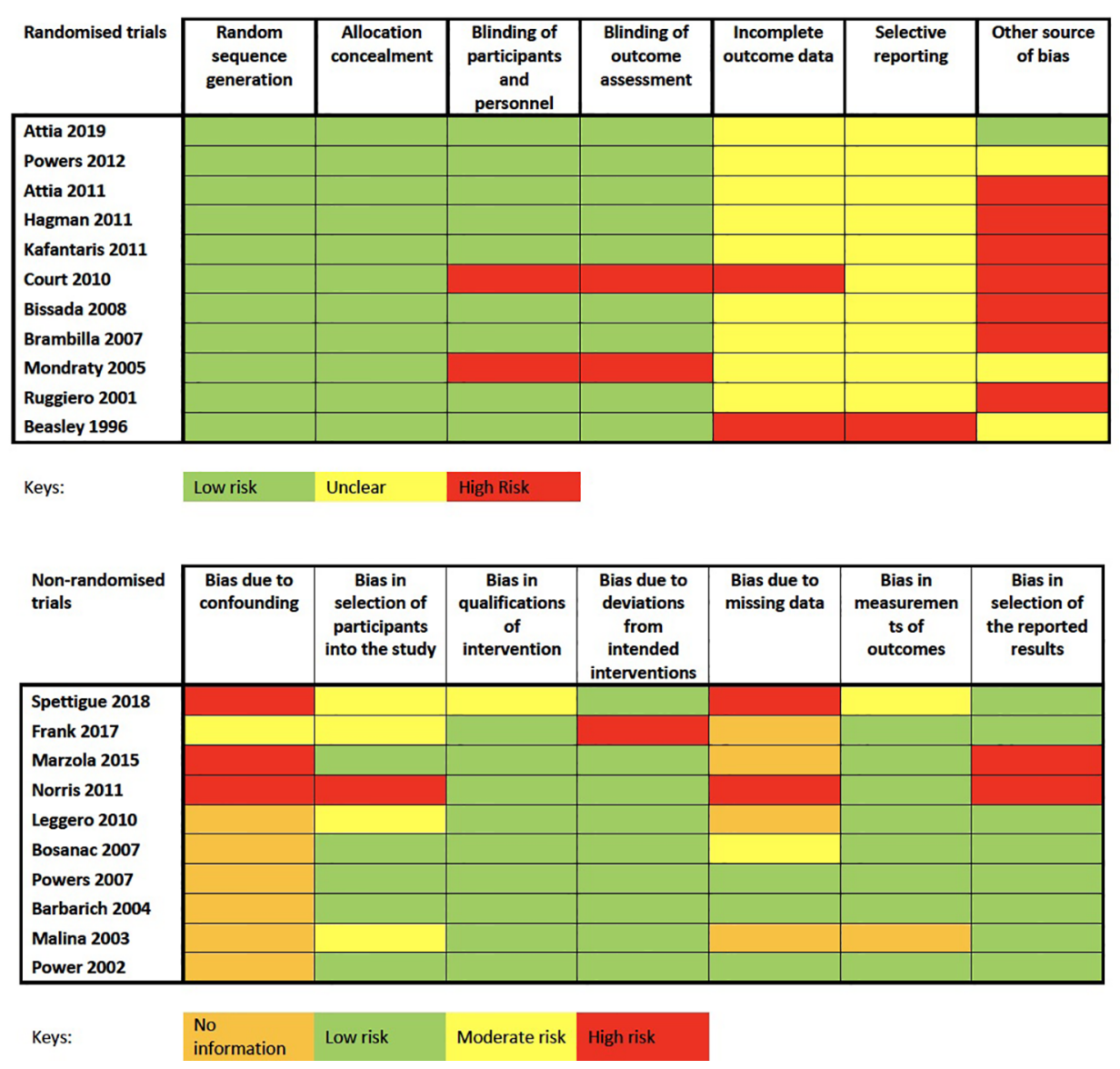

FIGURE 2 | Cochrane Collaboration's tool for assessing risk of bias for randomized trials and Risk of Bias in Non-randomized Studies-of Interventions (ROBINS-I) assessment tool for non-randomized studies.

\section{CONCLUSION}

Our study echoes the need to conduct high-quality psychopharmacological studies in people with AN. There are severe major limitations in the quality of the studies included in our meta-analysis. Future psychopharmacological studies for AN need to be large-scale and involve multiple centres while including qualitative feedback from patients and patientrelated outcomes to address these limitations. Involving patients and their families in the co-design process will potentially lead to better engagement and lower dropout rates. Overall, patient-related factors may explain drop-out rates in psychopharmacological trials even better than drug-related factors and side effects.

\section{AUTHOR CONTRIBUTIONS}

All authors were involved in the conception of the study. CK and JT designed the protocol for this analysis. CK performed the statistical analysis. CK and LE wrote the manuscript. All authors reviewed/edited the manuscript.

\section{FUNDING}

CK, JT, and HH are part funded by the NIHR Mental Health Biomedical Research Centre at South London and Maudsley NHS Foundation Trust and King's College London. CK has also previously received salary support from Novo Nordisk UK Research Foundation and Marie Curie Fellowship. The views expressed are those of the authors and not necessarily those of the NHS, the NIHR or the Department of Health.

\section{ACKNOWLEDGMENTS}

We would also like to thank Paul Lee, librarian at Lambeth Hospital of South London and Maudsley NHS Foundation Trust, for his helpful contribution with the literature search. 


\section{REFERENCES}

1. Steinhausen H-C. The Outcome of Anorexia Nervosa in the 20th Century. Am J Psychiatry (2002) 159:1284-93. doi: 10.1176/appi.ajp.159.8.1284

2. Eddy KT, Tabri N, Thomas JJ, Murray HB, Keshaviah A, Hastings E, et al. Recovery From Anorexia Nervosa and Bulimia Nervosa at 22-Year Follow-Up. J Clin Psychiatry (2017) 78:184-9. doi: 10.4088/ JCP. $15 \mathrm{~m} 10393$

3. Holland J, Hall N, Yeates DGR, Goldacre M. Trends in hospital admission rates for anorexia nervosa in Oxford (1968-2011) and England (1990-2011): database studies. J R Soc Med (2016) 109:59-66. doi: 10.1177/ 0141076815617651

4. NICE. National Clinical Practice Guideline: eating disorders: core interventions in the treatment and management of anorexia nervosa, bulimia nervosa, and related eating disorders. Natl Inst Clin Excell (2004) 9:2015.

5. NICE. (2018). Eating disorders: recognition and treatment., Available at: https://www.nice.org.uk/guidance/ng69.

6. Murray SB, Quintana DS, Loeb KL, Griffiths S, Le Grange D. Treatment outcomes for anorexia nervosa: a systematic review and meta-analysis of randomized controlled trials. Psychol Med (2018), 49(4):535-44. doi: 10.1017/ S0033291718002088

7. Hay PJ, Touyz S, Claudino AM, Lujic S, Smith CA, Madden S. Inpatient versus outpatient care, partial hospitalisation and waiting list for people with eating disorders. Cochrane Database Syst Rev (2019) 1:CD010827. doi: 10.1002/14651858.CD010827.pub2

8. Beykloo M Y, Nicholls D, Simic M, Brauer R, Mills E, Wong ICK. Survey on self-reported psychotropic drug prescribing practices of eating disorder psychiatrists for the treatment of young people with anorexia nervosa. BMJ Open (2019) 9:e031707. doi: 10.1136/bmjopen-2019-031707

9. Kishi T, Kafantaris V, Sunday S, Sheridan EM, Correll CU. Are antipsychotics effective for the treatment of anorexia nervosa? Results from a systematic review and meta-analysis. J Clin Psychiatry (2012) 73:e757-66. doi: 10.4088/ JCP.12r07691

10. Lebow J, Sim LA, Erwin PJ, Murad MH. The effect of atypical antipsychotic medications in individuals with anorexia nervosa: a systematic review and meta-analysis. Int J Eat Disord (2013) 46:332-9. doi: 10.1002/eat.22059

11. de Vos J, Houtzager L, Katsaragaki G, van de Berg E, Cuijpers P, Dekker J. Meta analysis on the efficacy of pharmacotherapy versus placebo on anorexia nervosa. J Eat Disord (2014) 2:27. doi: 10.1186/s40337-014-0027-x

12. Dold M, Aigner M, Klabunde M, Treasure J, Kasper S. Second-generation antipsychotic drugs in anorexia nervosa: a meta-analysis of randomized controlled trials. Psychother Psychosom (2015) 84:110-6. doi: 10.1159/ 000369978

13. Chaplain S, Taylor M. Drug points: second generation antipsychotics. Prescriber (2014) 14:12-21. doi: 10.1002/psb.1269

14. Watson HJ, Yilmaz Z, Thornton LM, Hübel C, Coleman JRI, Gaspar HA, et al. Genome-wide association study identifies eight risk loci and implicates metabo-psychiatric origins for anorexia nervosa. Nat Genet (2019) 51:120714. doi: 10.1038/s41588-019-0439-2

15. Himmerich H, Joaquim M, Bentley J, Kan C, Dornik J, Treasure J, et al. Psychopharmacological options for adult patients with anorexia nervosa: the patients' and carers' perspectives. CNS Spectr (2017) (4):251-52. doi: 10.1017/ S1092852917000529

16. Cuijpers P. Targets and outcomes of psychotherapies for mental disorders: an overview. World Psychiatry (2019) 18:276-85. doi: 10.1002/wps.20661

17. Higgins JPT, Altman DG, Gotzsche PC, Juni P, Moher D, Oxman AD, et al. The Cochrane Collaboration's tool for assessing risk of bias in randomised trials. BMJ (2011) 343:d5928-8. doi: 10.1136/bmj.d5928

18. Morgan RL, Thayer KA, Santesso N, Holloway AC, Blain R, Eftim SE, et al. Evaluation of the risk of bias in non-randomized studies of interventions (ROBINS-I) and the 'target experiment' concept in studies of exposures: Rationale and preliminary instrument development. Environ Int (2018) 120:382-7. doi: 10.1016/j.envint.2018.08.018

19. Viechtbauer W. Conducting meta-analyses in $\mathrm{R}$ with the metafor package. J Stat Softw (2010) 36:1-48. doi: 10.18637/jss.v036.i03
20. Barendregt JJ, Doi SA, Lee YY, Norman RE, Vos T. Meta-analysis of prevalence. J Epidemiol Community Health (2013) 67:974-8. doi: 10.1136/ jech-2013-203104

21. Freeman MF, Tukey JW. Transformations related to the angular and the square root. Ann Math Stat (1950) 21:607-11. doi: 10.1214/aoms/1177729756

22. Miller J. The inverse of the Freeman-Tukey double arcsine transformation. Am Stat (1978) 32:138. doi: 10.2307/2682942

23. Cochran WG. The comparison of percentages in matched samples. Biometrika (1950) 37:256-66. doi: 10.1093/biomet/37.3-4.256

24. Begg CB, Mazumdar M. Operating characteristics of a rank correlation test for publication bias. Biometrics (1994) 50:1088-101. doi: 10.2307/2533446

25. Brambilla F, Garcia CS, Fassino S, Daga GA, Favaro A, Santonastaso P, et al. Olanzapine therapy in anorexia nervosa: psychobiological effects. Int Clin Psychopharmacol (2007) 22:197-204. doi: 10.1097/YIC.0b013e328080ca31

26. Brambilla F, Monteleone P, Maj M. Olanzapine-induced weight gain in anorexia nervosa: involvement of leptin and ghrelin secretion? Psychoneuroendocrinology (2007) 32:402-6. doi: 10.1016/ j.psyneuen.2007.02.005

27. Bianco G, Claps M, Marinucci S, Montecchi F. Use of risperidone in adolescent anorexia nervosa. Ital J Psychiatry Behav Sci (2000) 10:50-2.

28. Attia E, Steinglass JE, Walsh BT, Wang Y, Wu P, Schreyer C, et al. Olanzapine Versus Placebo in Adult Outpatients With Anorexia Nervosa: A Randomized Clinical Trial. Am J Psychiatry (2019) 176:449-56. doi: 10.1176/ appi.ajp.2018.18101125

29. Spettigue W, Norris ML, Maras D, Obeid N, Feder S, Harrison ME, et al. Evaluation of the Effectiveness and Safety of Olanzapine as an Adjunctive Treatment for Anorexia Nervosa in Adolescents: An Open-Label Trial. J Can Acad Child Adolesc Psychiatry (2018) 27:197-208.

30. Frank GK, Shott ME, Hagman JO, Schiel MA, DeGuzman MC, Rossi B. The partial dopamine D2 receptor agonist aripiprazole is associated with weight gain in adolescent anorexia nervosa. Int J Eat Disord (2017) 50:447-50. doi: 10.1002/eat.22704

31. Marzola E, Desedime N, Giovannone C, Amianto F, Fassino S, Abbate-Daga G. Atypical antipsychotics as augmentation therapy in anorexia nervosa. PloS One (2015) 10:e0125569. doi: 10.1371/journal.pone.0125569

32. Powers PS, Klabunde M, Kaye W. Double-blind placebo-controlled trial of quetiapine in anorexia nervosa. Eur Eat Disord Rev (2012) 20:331-4. doi: 10.1002/erv.2169

33. Attia E, Kaplan AS, Walsh BT, Gershkovich M, Yilmaz Z, Musante D, et al. Olanzapine versus placebo for out-patients with anorexia nervosa. Psychol Med (2011) 41:2177-82. doi: 10.1017/S0033291711000390

34. Hagman J, Gralla J, Sigel E, Ellert S, Dodge M, Gardner R, et al. A doubleblind, placebo-controlled study of risperidone for the treatment of adolescents and young adults with anorexia nervosa: a pilot study. J Am Acad Child Adolesc Psychiatry (2011) 50:915-24. doi: 10.1016/j.jaac.2011.06.009

35. Kafantaris V, Leigh E, Hertz S, Berest A, Schebendach J, Sterling WM, et al. A Placebo-Controlled Pilot Study of Adjunctive Olanzapine for Adolescents with Anorexia Nervosa. J Child Adolesc Psychopharmacol (2011) 21:207-12. doi: 10.1089/cap.2010.0139

36. Norris ML, Spettigue W, Buchholz A, Henderson KA, Gomez R, Maras D, et al. Olanzapine Use for the Adjunctive Treatment of Adolescents with Anorexia Nervosa. J Child Adolesc Psychopharmacol (2011) 21:213-20. doi: 10.1089/cap.2010.0131

37. Court A, Mulder C, Kerr M, Yuen HP, Boasman M, Goldstone S, et al. Investigating the effectiveness, safety and tolerability of quetiapine in the treatment of anorexia nervosa in young people: a pilot study. J Psychiatr Res (2010) 44:1027-34. doi: 10.1016/j.jpsychires.2010.03.011

38. Leggero C, Masi G, Brunori E, Calderoni S, Carissimo R, Maestro S, et al. LowDose Olanzapine Monotherapy in Girls with Anorexia Nervosa, Restricting Subtype: Focus on Hyperactivity. J Child Adolesc Psychopharmacol (2010) 20:127-33. doi: 10.1089/cap.2009.0072

39. Bissada H, Tasca GA, Barber AM, Bradwejn J. Olanzapine in the Treatment of Low Body Weight and Obsessive Thinking in Women With Anorexia Nervosa: A Randomized, Double-Blind, Placebo-Controlled Trial. Am J Psychiatry (2008) 165:1281-8. doi: 10.1176/appi.ajp.2008.07121900

40. Bosanac P, Kurlender S, Norman T, Hallam K, Wesnes K, Manktelow T, et al. An open-label study of quetiapine in anorexia nervosa. Hum Psychopharmacol Clin $\operatorname{Exp}$ (2007) 22:223-30. doi: 10.1002/hup.845 
41. Powers PS, Bannon Y, Eubanks R, McCormick T. Quetiapine in anorexia nervosa patients: an open label outpatient pilot study. Int J Eat Disord (2007) 40:21-6. doi: 10.1002/eat.20325

42. Mondraty N, Birmingham CL, Touyz S, Sundakov V, Chapman L, Beumont P. Randomized controlled trial of olanzapine in the treatment of cognitions in anorexia nervosa. Australas Psychiatry (2005) 13:72-5. doi: 10.1080/j.14401665.2004.02154.x

43. Barbarich NC, McConaha CW, Gaskill J, La Via M, Frank GK, Achenbach S, et al. An open trial of olanzapine in anorexia nervosa. J Clin Psychiatry (2004) 65:1480-2. doi: 10.4088/JCP.v65n1106

44. Malina A, Gaskill J, McConaha C, Frank GK, LaVia M, Scholar L, et al. Olanzapine treatment of anorexia nervosa: a retrospective study. Int $J$ Eat Disord (2003) 33:234-7. doi: 10.1002/eat.10122

45. Powers PS, Santana CA, Bannon YS. Olanzapine in the treatment of anorexia nervosa: an open label trial. Int J Eat Disord (2002) 32:146-54. doi: 10.1002/ eat. 10084

46. Ruggiero GM, Laini V, Mauri MC, Ferrari VM, Clemente A, Lugo F, et al. A single blind comparison of amisulpride, fluoxetine and clomipramine in the treatment of restricting anorectics. Prog Neuropsychopharmacol Biol Psychiatry (2001) 25:1049-59. doi: 10.1016/s0278-5846(01)00174-9

47. Beasley CM, Sanger T, Satterlee W, Tollefson G, Tran P, Hamilton S. Olanzapine versus placebo: results of a double-blind, fixed-dose olanzapine trial. Psychopharmacol (Berl) (1996) 124:159-67. doi: 10.1007/ bf02245617
48. Kemmler G, Hummer M, Widschwendter C, Fleischhacker WW. Dropout rates in placebo-controlled and active-control clinical trials of antipsychotic drugs. Arch Gen Psychiatry (2005) 62:1305. doi: 10.1001/archpsyc.62.12.1305

49. Linardon J, Hindle A, Brennan L. Dropout from cognitive-behavioral therapy for eating disorders: a meta-analysis of randomized, controlled trials. Int J Eat Disord (2018). 51(5):381-91. doi: 10.1002/eat.22850

50. Costa MB, Melnik T. Effectiveness of psychosocial interventions in eating disorders: an overview of Cochrane systematic reviews. Einstein (Sao Paulo) (2016) 14:235-77. doi: 10.1590/S1679-45082016RW3120

51. Linardon J, Fitzsimmons-Craft EE, Brennan L, Barillaro M, Wilfley DE. Dropout from interpersonal psychotherapy for mental health disorders: a systematic review and meta-analysis. Psychother Res (2019) 29:870-81. doi: 10.1080/10503307.2018.1497215

Conflict of Interest: The authors declare that the research was conducted in the absence of any commercial or financial relationships that could be construed as a potential conflict of interest.

Copyright (c) 2020 Kan, Eid, Treasure and Himmerich. This is an open-access article distributed under the terms of the Creative Commons Attribution License (CC BY). The use, distribution or reproduction in other forums is permitted, provided the original author(s) and the copyright owner(s) are credited and that the original publication in this journal is cited, in accordance with accepted academic practice. No use, distribution or reproduction is permitted which does not comply with these terms. 


\section{APPENDIX 1 EXHAUSTIVE LIST OF KEYWORDS FOR SEARCH}

1. 'anorexi' $\mathrm{OR}$ 'anorexia nervosa'

2. 'atypical antipsychotic' OR 'atypical neuroleptic' OR 'second generation

antipsychotic' OR 'second generation neuroleptic' OR amisulpride OR

aripiprazole OR asenapine OR brexpiprazole OR cariprazine OR clozapine OR

lurasidone OR 'lurasidone hydrochloride' OR olanzapine OR paliperidone OR

'paliperidone palmitate' OR primavenserin OR quetiapine OR 'quetiapine

fumarate' OR remoxipride OR risperidone OR sertindole OR ziprasidone.

3. metaboli^ OR BMI OR 'body mass index' OR obesity OR hba1c OR 'blood

pressure' OR hypertension OR cholesterol OR QTc OR prolactin OR

hyperglycemi* OR diabetes OR 'weight gain' OR 'weight increase' OR 'metabolic

side effects of drugs and substances' OR 'metabolic diseases' OR 'metabolic

syndrome' OR 'weight gain - drug effects' OR 'metabolic networks and

pathways - drug effects' OR 'body weight - drug effects

4. 1 AND 2 AND 3

5. Limit 4 to humans + English language 